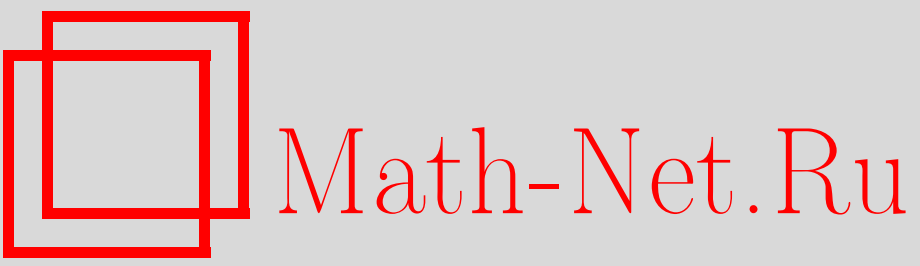

П. А. Колгушкин, Р. Р. Садыков, Классификация простых мультиростков кривых, УМН, 2001, том 56, выпуск 6, 153-154

DOI: https://doi.org/10.4213/rm463

Использование Общероссийского математического портала Math-Net.Ru подразумевает, что вы прочитали и согласны с пользовательским соглашением

http://www.mathnet.ru/rus/agreement

Параметры загрузки:

IP: 3.89 .185 .249

26 апреля 2023 г., 13:23:29 


\title{
КЛАССИФИКАЦИЯ ПРОСТЫХ МУЛЬТИРОСТКОВ КРИВЫХ
}

\author{
П. А. КолгУшкин, Р. Р. САдыков
}

Мультиросток кривой в $\mathbb{C}^{n}$ - это множество $F=\left(f_{1}, \ldots, f_{k}\right)$ ростков аналитических отображений $f_{i}:(\mathbb{C}, 0) \rightarrow\left(\mathbb{C}^{n}, 0\right)$, где $\operatorname{Im} f_{i} \cap \operatorname{Im} f_{j}=\{0\}$ для $i \neq j\left(f_{1}, \ldots, f_{k}\right.$ назьваются компонентами мультростка). Мултиросток является локалшной параметризацией ростка приводимой кривой в нуле. Мултиросток называется простым, если у него имеется окрестность, пересекающая лишь конечное число орбит действия группы $A$ право-левых координатных замен.

Мы классифицируем стабилшно простые особенности мульиростков в конечномерных комплексных пространствах произвольной размерности относительно стабильной эквивалентности. Определения и мотивировку задачи см. в [1]

Обозначим через $G_{n}$ мультиросток, состоящий из первых $n$ координатных осей в $\mathbb{C}^{N}$, через $\left(t^{m} \times k\right)$ - неприводимую кривую вида $\left(t^{m}, \ldots, t^{m}\right)(k$ мономов).

ТеОРема. Каждый стабильно простой мультиростож стабильно әквивалентен одному и только одному мультиростку из следующего списка $(m, n, k$ u $l$ - натуральнье числа):

1. Пары кривых с неособой первой компонентой. Здесь первая компонента равна $(t, 0)$. В списке мы указываем только вторую компоненту.

1.1. Мультиростки с обеими неособыми компонентами:

1. $(0, t) ; \mathbf{2} .\left(t, t^{k}\right), k>1$.

1.2. Мультиростки со второй компонентой кратности два:

1. $\left(t^{2}, t^{2 m+1}\right) ;$ 2. $\left(t^{2}, t^{2 m+1}+t^{2 n}\right), m<n<2 m ; 3 .\left(t^{2}, t^{2 m+1}, t^{2 n}\right), m<n \leqslant 2 m$; 4. $\left(t^{2}, t^{2 m+1}+t^{2 n}, t^{2 s}\right), m<n<s \leqslant 2 m ;$ 5. $\left(t^{2}, t^{2 n}+t^{2 m+1}\right), n \leqslant m ; \mathbf{6} \cdot\left(t^{2}, t^{2 n}, t^{2 m+1}\right)$, $n \leqslant m ; \mathbf{7} \cdot\left(t^{2}, t^{2 n}+t^{2 m+1}, t^{2 s+1}\right), n \leqslant m<s<m+n ; \mathbf{8} \cdot\left(t^{2 r+1}, t^{2}\right) ; \mathbf{9} \cdot\left(0, t^{2}, t^{2 r+1}\right)$;

1.3. Мультиростки с 3 -струей $\left((t, 0),\left(0, t^{3}\right)\right)$ :

1. $\left(t^{3 m+1}, t^{3}\right) ; \mathbf{2} \cdot\left(t^{3 m+1}, t^{3}, t^{3 n+2}\right), m \leqslant n \leqslant 2 m ;$ 3. $\left(t^{3 m+1}+t^{3 n+2}, t^{3}\right), m \leqslant n<2 m ;$ 4. $\left(t^{3 m+1}+t^{3 n+2}, t^{3}, t^{3 l+2}\right), m \leqslant n<l \leqslant 2 m ;$ 5. $\left(t^{3 m+2}, t^{3}\right) ; \mathbf{6} \cdot\left(t^{3 m+2}, t^{3}, t^{3 n+1}\right), m<n \leqslant$ $2 m+1 ; 7 \cdot\left(t^{3 m+2}+t^{3 n+1}, t^{3}\right), m<n \leqslant 2 m ; 8 \cdot\left(t^{3 m+2}+t^{3 n+1}, t^{3}, t^{3 l+2}\right), m<n<l \leqslant$ $2 m+1 ;$ 9. $\left(0, t^{3}, t^{3 m+1}\right) ; \mathbf{1 0} .\left(t^{3 n+2}, t^{3}, t^{3 m+1}\right), m \leqslant n<2 m ; 11 .\left(t^{3 l+2}, t^{3}, t^{3 m+1}+t^{3 n+2}\right)$, $m \leqslant n \leqslant l<2 m$, кроме $n=l=2 m-1 ; \mathbf{1 2} .\left(0, t^{3}, t^{3 m+2}\right) ; \mathbf{1 3 .}\left(t^{3 l+1}, t^{3}, t^{3 m+2}+t^{3 n+1}\right), m<$ $n \leqslant l \leqslant 2 m$, кроме $n=l=2 m ; \mathbf{1 4} .\left(t^{3 n+1}, t^{3}, t^{3 m+2}\right), m<n \leqslant 2 m ; \mathbf{1 5} .\left(0, t^{3}, t^{3 m+1}, t^{3 n+2}\right)$, $m \leqslant n<2 m ; \mathbf{1 6} .\left(0, t^{3}, t^{3 m+1}+t^{3 n+2}\right), m \leqslant n<2 m-1 ; \mathbf{1 7} \cdot\left(0, t^{3}, t^{3 m+1}+t^{3 n+2}, t^{3 l+2}\right)$, $m \leqslant n<l<2 m ;$ 18. $\left(0, t^{3}, t^{3 m+2}, t^{3 n+1}\right), m<n \leqslant 2 m ; 1$ 19. $\left(0, t^{3}, t^{3 m+2}+t^{3 n+1}\right), m<n<$ $2 m ;$ 20. $\left(0, t^{3}, t^{3 m+2}+t^{3 n+1}, t^{3 l+1}\right), m<n<l \leqslant 2 m$.

1.4. Мультиростки с 3 -струей $\left((t, 0),\left(t^{3}, 0\right)\right)$ :

1. $\left(t^{3}, t^{4}\right) ;$ 2. $\left(t^{3}, t^{4}, t^{5}\right) ;$ 3. $\left(t^{3}, t^{4}, t^{5}, t^{6}\right) ;$ 4. $\left(t^{3}, t^{4}+t^{6}\right) ;$ 5. $\left(t^{3}, t^{4}+t^{6}, t^{9}\right) ; 6$. $\left(t^{3}, t^{4}, t^{6}\right)$ 7. $\left(t^{3}, t^{4}, t^{9}\right) ;$ 8. $\left(t^{3}, t^{5}, t^{6}\right) ; 9 .\left(t^{3}, t^{5}, t^{6}+t^{7}\right) ; 10 .\left(t^{3}, t^{5}, t^{6}, t^{7}\right) ; 11 .\left(t^{3}, t^{5}+t^{6}, t^{7}\right) ; 12 .\left(t^{3}, t^{5}+\right.$ $\left.t^{6}, t^{7}, t^{9}\right) ; 13 .\left(t^{3}, t^{5}+t^{6}, t^{9}\right) ; 14 .\left(t^{3}, t^{5}, t^{7}\right) ; 15 .\left(t^{3}, t^{5}, t^{7}, t^{9}\right) ; 1$ 16. $\left(t^{3}, t^{5}, t^{9}\right) ; 17 .\left(t^{3}, t^{5}+\right.$ $\left.t^{6}, t^{12}\right)$ 18. $\left(t^{3}, t^{5}+t^{6}\right)$; 19. $\left(t^{3}, t^{5}\right) ; 20 .\left(t^{3}, t^{5}, t^{12}\right) ; 21 .\left(t^{3}, t^{5}+t^{9}\right) ; 22$. $\left(t^{3}, t^{5}+t^{9}, t^{12}\right)$; 23. $\left(t^{3}, t^{6}, t^{7}, t^{8}\right)$

1.5 Мультиростки с 4-струями $\left((t, 0),\left(0, t^{4}\right)\right)$ и $\left((t, 0),\left(t^{4}, 0\right)\right)$ :

1. $\left(t^{5}, t^{4}, t^{6}, t^{7}\right) ; 2 .\left(t^{6}, t^{4}, t^{5}, t^{7}\right) ; 3 .\left(0, t^{4}, t^{5}, t^{7}\right) ; 4$. $\left(0, t^{4}, t^{5}, t^{6}\right) ; 5$. $\left(0, t^{4}, t^{5}, t^{6}, t^{7}\right) ; 6 .\left(t^{7}\right.$, $\left.t^{4}, t^{5}, t^{6}\right) ; 7$. $\left(0, t^{4}, t^{6}, t^{7}, t^{9}\right) ; 8 .\left(0, t^{4}, t^{6}, t^{7}\right) ; 9 .\left(t^{9}, t^{4}, t^{6}, t^{7}\right) ; 10 .\left(t^{4}, t^{5}, t^{6}, t^{7}\right) ; 11 .\left(t^{4}, t^{5}, t^{6}\right.$, $\left.t^{7}, t^{8}\right)$.

1.6. Мультиростки с 5 -струей $\left((t, 0),\left(0, t^{5}\right)\right)$ :

Работа выполнена при частичной поддержке Российского фонда фундаментальных исследований (грант № 01-01-00739) и NWD-RFBR 047.008.005. 
1. $\left(t^{9}, t^{5}, t^{6}, t^{7}, t^{8}\right) ; 2 .\left(0, t^{5}, t^{6}, t^{7}, t^{8}\right) ;$ 3. $\left(0, t^{5}, t^{6}, t^{7}, t^{8}, t^{9}\right) ; 4 \cdot\left(t^{8}, t^{5}, t^{6}, t^{7}, t^{9}\right) ; \mathbf{5}$. $\left(0, t^{5}\right.$, $\left.t^{6}, t^{7}, t^{9}\right)$.

2. Пары кривых с обеими особыми компонентами.

2.1. Бесконечные серии: Здесь первая компонента равна $\left(t^{2}, t^{2 m+1}\right)$. Мы указываем только вторую компоненту (здесь $m \leqslant n$ ).

1. $\left(t^{2 n+1}, t^{2}\right) ; \mathbf{2} .\left(t^{2 n+1}, t^{2}, t^{2 n+3}\right) ;$ 3. $\left(0, t^{2}, t^{2 n+1}\right) ;$ 4. $\left(t^{2 n+1}, 0, t^{2}\right) ;$ 5. $\left(0, t^{2 n+1}, t^{2}\right) ; \mathbf{6}$. $(0$, $\left.0, t^{2}, t^{2 n+1}\right)$.

2.2. Индивидуальные особенности: Здесь первая компонента равна $\left(t^{2}, t^{3}\right)$.

1. $\left(t^{2}, 0, t^{3}, t^{4}\right) ; \mathbf{2 .}\left(t^{2}, 0, t^{3}\right) ; \mathbf{3 .}\left(0,0, t^{3}, t^{4}, t^{5}\right) ; \mathbf{4 .}\left(0, t^{5}, t^{3}, t^{4}\right) ; \mathbf{5 .}\left(t^{5}, 0, t^{3}, t^{4}\right) ; \mathbf{6} .\left(0,0, t^{3}, t^{4}\right)$; 7. $\left(0, t^{4}, t^{3}, t^{5}\right) ; \mathbf{8 .}\left(t^{4}, 0, t^{3}, t^{5}\right) ; \mathbf{9 .}\left(0,0, t^{3}, t^{5}, t^{7}\right) ; 10 .\left(0, t^{7}, t^{3}, t^{5}\right) ; 11 .\left(t^{7}, 0, t^{3}, t^{5}\right) ; 12 .(0,0$, $\left.t^{3}, t^{5}\right)$.

3. Мультиростки с неособыми компонентами:

1. $G_{n} ; \mathbf{2} . G_{n},(t \times k, 0, \ldots, 0), 1<k \leqslant n ; \mathbf{3 .} G_{n},\left(t, t^{m} \times k, 0, \ldots, 0\right), 1 \leqslant k<n, m>1$; 4. $G_{n},\left(t, 0 \times(n-1), t^{m}\right), m>1 ; \mathbf{5} \cdot\left(t_{1}, 0,0\right),\left(t_{2}, t_{2}^{2}, 0\right),\left(t_{3}, 0, t_{3}^{2}\right)$.

4. Мультиростки с одной особой компонентой.

4.1 Серии, содержащие произвольное число неособых компонент: Здесь неособая часть мульиростка равна $G_{n}, n \geqslant 2$. Мы указываем только особую компоненту.

1. $\left(0 \times n, t^{2}, t^{2 m+1}\right) ; \mathbf{2} .\left(t^{2 m+1} \times k, 0 \times(n-k), t^{2}\right), 1 \leqslant k \leqslant n ; \mathbf{3} .\left(t^{2} \times k, 0 \times(n-k), t^{3}\right)$, $1<k \leqslant n ;$ 4. $\left(t^{2}, 0 \times(n-1), t^{3}, t^{4}\right) ; \mathbf{5} .\left(t^{2}, t^{4} \times k, 0 \times(n-k-1), t^{3}\right), 0 \leqslant k<n ; \mathbf{6} .(0 \times n$, $\left.t^{3}, t^{4}, t^{5}\right) ; 7 \cdot\left(t^{5} \times k, 0 \times(n-k), t^{3}, t^{4}\right), 0 \leqslant k \leqslant n ; 8 .\left(t^{4} \times k, 0 \times(n-k), t^{3}, t^{5}\right), 0 \leqslant k \leqslant n$; 9. $\left(0 \times n, t^{3}, t^{5}, t^{7}\right) ; \mathbf{1 0} .\left(t^{7} \times k, 0 \times(n-k), t^{3}, t^{5}\right), 1 \leqslant k \leqslant n$.

4.2. Бесконечные серии, содержащие две неособые компоненты: Здесь неособая часть мультиростка равна $G_{2}$. Мы указываем только особую компоненту.

1. $\left(t^{2}, t^{2}, t^{2 m+1}\right), m \geqslant 2 ; \mathbf{2} \cdot\left(t^{2}, t^{2}+t^{2 m+1}, t^{2 m+3}\right), m \geqslant 1 ; 3 \cdot\left(t^{2}, t^{2}+t^{2 m+1}\right), m \geqslant 1$; 4. $\left(t^{2}, 0, t^{2 m+1}, t^{2 n}\right), m<n \leqslant 2 m ; \mathbf{5} .\left(t^{2}, t^{2 n}, t^{2 m+1}+t^{2 n}\right), m<n<2 m ; \mathbf{6} .\left(t^{2}, t^{2 n}, t^{2 m+1}\right)$, $m<n \leqslant 2 m ;$ 7. $\left(t^{2}, 0, t^{2 m+1}+t^{2 n}, t^{2 l}\right), m<n<l \leqslant 2 m ; 8 .\left(t^{2}, t^{2 l}, t^{2 m+1}+t^{2 n}\right), m<n<l \leqslant$ $2 m ; \mathbf{9} .\left(t^{2}, 0, t^{2 m+1}+t^{2 n}\right), m<n<2 m ; \mathbf{1 0} .\left(t^{2}, 0, t^{2 m+1}\right) ; \mathbf{1 1} .\left(t^{2}, t^{2 m+1}, t^{2 n}\right), m<n \leqslant 2 m+$ 1; 12. $\left(t^{2}, t^{2 m+1}+t^{2 n}, t^{2 l}\right), n<l \leqslant 2 m+1 ; 13 .\left(t^{2}, t^{2 m+1}+t^{2 n}\right), m<n \leqslant 2 m$; 14. $\left(t^{2}, t^{2 m+1}\right)$; 15. $\left(t^{2}, 0, t^{2 m}, t^{2 n+1}\right), 1<m \leqslant n ; \mathbf{1 6} .\left(t^{2}, t^{2 n+1}, t^{2 m}\right), m \leqslant n ; 17 .\left(t^{2}, 0, t^{2 m}+t^{2 n+1}, t^{2 l+1}\right)$, $m \leqslant n<l<m+n ; \mathbf{1 8} .\left(t^{2}, t^{2 l+1}, t^{2 m}+t^{2 n+1}\right), m \leqslant n \leqslant l<m+n ; \mathbf{1 9} .\left(t^{2}, 0, t^{2 m}+t^{2 n+1}\right)$, $1<m \leqslant n ;$ 20. $\left(t^{2}, t^{2 m}, t^{2 n+1}\right), 1<m \leqslant n ; \mathbf{2 1} .\left(t^{2}, t^{2 m}+t^{2 n+1}, t^{2 l+1}\right), m \leqslant n<l \leqslant m+n$; 22. $\left(t^{2}, t^{2 m}+t^{2 n+1}\right), 1<m \leqslant n$.

4.3. Индивидуальные особенности: Здесь неособая часть мультиростка равна $G_{2}$.

1. $\left(t^{3}, t^{3}, t^{4}, t^{5}\right) ;$ 2. $\left(t^{3}, 0, t^{4}, t^{5}, t^{6}\right) ;$ 3. $\left(t^{3}, t^{6}, t^{4}, t^{5}\right) ;$ 4. $\left(t^{3}, 0, t^{4}, t^{5}\right) ;$ 5. $\left(0,0, t^{4}, t^{5}, t^{6}, t^{7}\right)$; 6. $\left(t^{7}, t^{7}, t^{4}, t^{5}, t^{6}\right) ; 7 \cdot\left(t^{7}, 0, t^{4}, t^{5}, t^{6}\right) ; \mathbf{8 .}\left(0,0, t^{4}, t^{5}, t^{6}\right) ; \mathbf{9 .}\left(t^{6}, t^{6}, t^{4}, t^{5}, t^{7}\right) ; 10 .\left(t^{6}, 0, t^{4}, t^{5}, t^{7}\right)$; 11. $\left(0,0, t^{4}, t^{5}, t^{7}\right)$.

4.4. Серии с неособой частью $\left(\left(t_{1}, 0\right),\left(t_{2}, t_{2}^{2}\right)\right)$ :

1. $\left(0,0, t^{2}, t^{2 m+1}\right) ; \mathbf{2} .\left(0, t^{2 m+1}, t^{2}\right) ; \mathbf{3} \cdot\left(t^{2 m+1}, 0, t^{2}\right)$.

5. Мультиростки с двумя особыми и одной неособой компонентами. Первая и третья компоненты равны $(t, 0,0,0)$ и $\left(0,0, t_{2}^{2}, t_{2}^{3}\right)$ соответственно. В следующем ниже списке $m \geqslant 1$.

1. $\left(0, t_{1}^{2}, 0,0, t_{1}^{2 m+1}\right) ; \mathbf{2} \cdot\left(t_{1}^{2 m+1}, t_{1}^{2}, 0, t_{1}^{2 m+1}\right) ; \mathbf{3 .}\left(0, t_{1}^{2}, 0, t_{1}^{2 m+1}\right) ; \mathbf{4} \cdot\left(t_{1}^{2 m+1}, t_{1}^{2}, t_{1}^{2 m+1}, 0\right)$; 5. $\left(0, t_{1}^{2}, t_{1}^{2 m+1}, 0\right) ; \mathbf{6} \cdot\left(t_{1}^{2 m+1}, t_{1}^{2}, 0,0\right)$.

Доказательство, в основном, основано на методе полных трансверсалей, см. [2].

\section{СПИСОК ЛИТЕРАТУРЫ}

[1] В. И. Арнольд // Труды МИАН. 1999. Т. 226. С. 27-35. [2] J. W. Bruce, N. P. Kirk, A. A. du Plessis // Nonlinearity. 1997. V. 10. № 1. P. 253-275. 